\section{ITALIAN SOCIETY FOR GEOPHYSICS AND METEOROLOGY}

\section{FOURTH GENERAL ASSEMBLY}

$\mathrm{T}$ HE fourth General Assembly of the Società Italiana di Geofisica e Meteorologia (Genova) was held at the University of Genoa during April 5-8. The convention was opened by the president, Prof. M. Bossolasco, who welcomed the delegates to the Assembly. A large number of papers ranging over a wide number of topics was presented.

Prof. A. Defant (Innsbruck) delivered the opening lecture, on "Recent Developments in the Theory of Ocean Currents". Referring to the classical theory due to V. Ekman for a homogeneous ocean, he gave an account of the recent contribution due to $H$. Stommel and W. Munk, pointing out the objections directed to it and its limitation. He then discussed the theory of ocean currents due to G. Neumann, which uses as surface of reference that deduced for the Atlantic from the observations of the expedition Meteor. Prof. Defant showed that this new theory gives a possible explanation of the intensification of ocean currents on their west coasts. Reference was also made to some work by Charney on the formation of narrow currents on these coasts.

Prof. Perri (Turin) spoke on "The Slow Surface Wave and its Seismological Significance in a Limiting Case". This referred to a slow surface which was discovered by the author, the theory of which he developed in 1954. Prof. Perri recalled its main features and attributed the fact that the velocity of the wave is intermediate between that of the Rayleigh waves and Love waves to an abnormal dispersion.

Prof. A. Giao (Paris) presented a paper on "The Behaviour of the Advection Vector of the Perturbations of the Vertical Vorticity in the Free Atmosphere". After discussing the definition of the advection vector, Prof. Giao went on to consider applications to the atmosphere. By means of the observed temperature fields and wind between the equator and the poles (from sea-level to the $20-\mathrm{km}$. level) one can deduce the mean field of the advection vector of the perturbations and compare it with the mean wind in the field. This leads to the explanation of many empirical facts and gives the limits of the region of 'long waves'. The second part of the paper dealt with the derivation of a generalized equation for the vertical vorticity by a simple transformation of the equation of pressure variation in Prof. Giao's theory of perturbation.

"The Oscillations of the Natural Radioactivity of the Air in the Northern Alps, and Their Significance" was the title of a paper by Dr. R. Reiter (Munich). Dr. Reiter has found from many measurements of the air of the Zugspitze $(2,600 \mathrm{~m}$. above sea-level) that the greatest radioactivity is associated with southerly currents. This is considered to point to a dependence on the nature of the geological formations traversed by these winds, the formations containing granite and orthogneiss. Dr. Reiter also discussed the variations of the radioactivity of the air as a function of other meteorological factors.

A paper by Dr. C. Mennella (Naples) dealt with "The Reliability of the Presumed Normal Values of the Rainfall in Italy". Considering various series of rainfall measurements for the past hundred years or so, Dr. Mennella attempted to appraise the reliability of the mean values relative to quinquennial, decennial and longer periods. This enables one to estimate the degree of variability and instability and to deduce to what extent they are inadequate to represent the specific characteristics of the phenomena.

Dr. W. Hesse (Leipzig) spoke on "The Hydrogenic Balance of Plants" and on "The Results of Measurements made on Various Plants as Functions of Meteorological Factors". Dr. Hesse pointed out that the water system of cultivated plants is very complicated and is influenced by various external factors ; on the meteorological side, several methods have been evolved to assess evaporation and transpiration of plants. In the vegetation period 1950, 1953, 1954 and 1955, Dr. Hesse used the newly developed Klein lysimeter in Leipzig, and from 1954 onwards a transpirograph and a measuring apparatus consisting of six different Klein Iysimeters with which it was possible to assoss the water content of plants.

Prof. O. Burkard (Graz) proposed a formula for the moving ionization of the $\vec{F}_{2}$ layer in Europe. He showed that the squares of the critical frequencies $\left(f_{0} F_{2}\right)^{2}$ measured at European stations in the morning may be represented by the empirical formula:

$$
\left(f_{0} F_{2}\right)^{2}=K\left(\frac{\cos \chi}{\cos ^{2} \varphi}\right)^{x} \cos ^{2} \varphi
$$

where $\chi$ is the zenith angle of the Sun, $\varphi$ the geographical latitude of the station, $x$ depends on locality, $K$ is a constant depending on the locality and probably on the intensity of the solar ionizing radiation.

The communications from Prof. P. G. Piccardi and Dr. R. Cini (Florence) were presented by Prof. Bossolasco. These related to "The Action of an Electromagnetic Field of Frequency $10 \mathrm{kHz}$. on a Chemical Test" and "The Problem of the Influence of Atmospherics". Experiments showed that Prof. Piccardi's chemical test is influenced by an electromagnetic field of frequency $10 \mathrm{kHz}$. (kc./s.) and that it is probable that the variations observed during this test are due to variation in solar electromagnetic radiation as well as atmospherics due to lightning discharges.

Prof. V. Ferraro (London) gave an account of the present state of theories of magnetic storms. He pointed out that an atmospheric-current theory such as has been proposed by Vestine is unlikely to prove correct since on this theory it seems difficult to account for such distinctive phenomena as sudden commencements, auroral zones and other features which point rather to an influx of solar corpuscular streams in the upper atmosphere as the cause of these phenomena.

Dr. K. Rawer (Neuershausen b. Freiburg) gave an introductory talk to the general discussion on the International Geophysical Year. Dr. Rawer discussed in particular preparations made for the study of ionospheric recordings, absorption, 'winds', back scatter and whistlers. The discussion that followed related particularly to questions of interest to the Italian participants of the International Geophysical Year.

In a paper on "The Harmonic and Anharmonic Constants for the Tides at Palermo, Milazzo, Lipari and Cagliari", Dr. S. Polli (Trieste) said these are determined for periods of 26 months in the case of Palermo, and shorter periods for the other stations, by using the British Admiralty method developed by Doodson. The monthly and seasonal variations in 
the tides are determined separately, so as to examine any seasonal variations of the constants. A second paper dealt with wind-bores in the ports of Trieste and Venice; it appears that this wind-bore traverses the gulf of Venice without change of direction, lateral expansion and with little loss of speed.

Prof. H. Berg (Cologne) dealt first with "The Velocity Profile in Rivers" - an attempt to express the vertical velocity gradient of rivers by a logarithmic law using certain ideas of Prandt concerning turbulent flow over rough surfaces. In a second paper, on "Examples of Trivial Correlations in Solar Terrestrial Relationships", Prof. Berg examined two such examples: Farthing's experiment, which led him to conclude that a period of intense coronal radiation is followed by a penetration of cold air over the Earth; and an investigation by Abbott, who claimed that the solar constant is considerably lowered on days of intense geomagnetic storms. Prof. Berg showed that the results in both cases are only the outcome of faulty mathematical reasoning.

Prof. G. Berbesconi (Genoa) considered the "Partial and Total Representation of an Ellipsoid on the Sphere". Starting from the principle of the minimum energy of deformation of an elastic ellipsoidal geographical network, Prof. Berbesconi determines a relationship between the ellipsoid of gyration and the local spherical surface in the neighbourhood of the oscillation point up to a radius of $250 \mathrm{~km}$. He next examines various functions the extremals of which give a good representation of the whole ellipsoid on the sphere and define a new latitude which he considers useful for the construction of geographical globes.

Dr. I. Dagnino (Genoa) considered the "Frequency of Cyclones and the Distribution of Cloud in the Mediterranean", using the synoptic charts for all the days during the period 1946-55. For the case of the region of maximum frequency, Dr. Dagnino has examined and discussed the principal atmospheric and meteorological factors giving rise to these cyclones-in addition to the seasonal factors.

Prof. Bossolasco (Genoa) spoke on some work he has done with collaborators on "The Atmospheric Electric Potential in the Layers close to the Ground". The result of a series of observations (with Drs. Dagnino and Romagnone) to determine the variation of the atmospheric electric field near ground-level showed a decrease of the potential gradient from a height of $1 \mathrm{~m}$. to $2 \mathrm{~m}$., and a further decrease at a height of $3 \mathrm{~m}$. The variation at discrete potentiallevels confirms electrically the presence of a boundary layer near the ground. A second communication dealt with the "Measurement and Continuous Recording of the Salinity of the Atmosphere". The apparatus used for the purpose is one developed by F. Meda, based on the principle of collecting the radioactivity by passing the air through water.

Prof. M. Cutolo (Naples) spoke on "The Measurement and Behaviour of the Geomagnetic Field at the Lower Border of the E-layer'. He described how this can be effected by taking the mean of the frequencies of the two maxima in the gyro-interaction curve and pointed out that the measurements of the geomagnetic field would be very accurate. Prof. Cutolo also spoke on "The Effects of Demodulation in the Ionosphere" ; this method also provides an alternative method of measuring the geomagnetic field in the $E$-layer.

"Micropulsation in the Geomagnetic Horizontal Force and in Telluric Currents" was the subject of a paper by Dr. K. Burkhat (Munich). Dr. Burkhat pointed out that although the close connexion between geomagnetic variations and telluric currents has been known for more than a century, it has only recently been possible to decide which of the two phenomena is the cause and which the effect. At large distances from the electrodes, and in the case of small periods, the respective phases are in good accord. But for small periods (diurnal variation) and close to the electrodes, one finds a definite difference in the phase of the currents with respect to the geomagnetic variations. The difference in phase can be explained as due to the effect of resistance and reactance, since we now know that every telluric current connected with geomagnetic variation is due to induced currents. Dr. Burkhat then considered the statistics of pulsations and noted that the frequency curve for pulsations with periods of about 30 sec. coincides very nearly with the variation of maximum electron density in the $E$ - and $F_{1}$-layers.

Prof. M. Matschinski (Paris) next spoke on "Problems of Geochemistry". He confined himself to what he described as the geographical aspects of geochemistry-which problem he formulated as that of finding the relationship between the chemical and geographical traits of given regions. As an example, he considered volcanic regions in the form of ares; he recalled that he has already found a relationship between the density and the radius of curvature of the arcs. Prof. Matschinski showed several graphs with the view of demonstrating a statistical interdependence between geographical traits and certain chemical characteristics of rocks of the regions considered; for example, the mean percentages of the oxides of silicon, aluminium, calcium, magnesium, sodium, potassium and iron. These results were obtained from more than two thousand analyses.

The prize for meteorology offered by the Society was awarded to Prof. A. Giao and M. Ferreira for their paper "Introduzione alla climatologia dinamica dell'America Settendrionale, del Nord-Atlantico e dell'Europa". The following officers were elected for the period 1956-59 : President, Prof. M. Bossolasco, Council, Profs. Aliverti, Lovera and Ranzi ; Secretary, Dr. I. Dagnino. V. C. A. Ferraro

\section{MEMBRANE PHENOMENA}

\section{FARADAY SOCIETY DISCUSSION}

$\mathrm{T}$ THE Faraday Society held a general discussion on "Membrane Phenomena" in the University of Nottingham during April 10-12. Nearly two hundred scientific workers attended the discussion, the first of its kind to be held in Nottingham. The president, Mr. R. P. Bell, took the chair for the whole of the meeting, and the proceedings were opened by the sending of a message of greeting to Prof. F. G. Donnan, a founder-member and pastpresident of the Society, who is well known for his work in the field of membrane equilibria.

The discussion proper was preceded by the Spiers Memorial Lecture, given on this occasion by Prof. $T$. Teorell (Upsala), whose subject was "Transport Phenomena in Membranes". Prof. Teorell indicated how his own interest sprang originally from biochemical studies on hydrochloric acid in the stomach. $\mathrm{He}$ outlined structural studies on cell membranes, showing slides of striking electron micrographs taken 